\title{
Digitalisierung in Studium und Lehre als strategische Chance für Hochschulen
}

\author{
Strategie-, Struktur- und Kulturentwicklung gestalten
}

\section{Digitalisierung in Studium und Lehre als Chance ergreifen}

Die Digitalisierung ist in unserer Gesellschaft, Arbeitswelt und auch an den Hochschulen längst angekommen. Nicht zuletzt Hochschulleitungen erkennen zunehmend ihre Bedeutung für die Hochschulentwicklung und auch die Kultusministerkonferenz (KMK) hat kürzlich deutlich gemacht, dass „die Digitalisierung von Studium und Lehre [...] Gegenstand der strategischen Hochschulentwicklung“ (KMK 2019) ist. Die Hochschule als Lernwelt verändert sich grundlegend und die digitale Transformation der Hochschulbildung spielt dabei eine zentrale Rolle.

Übergreifend kann die Digitalisierung in Studium und Lehre als ein transformativer Prozess verstanden werden, der alle Aktivitäten der Hochschulen wesentlich beeinflusst. Die digitale Transformation durchdringt alle Strukturen, Orte, Formate und Ziele von Lehre, Lernen, Forschen und Arbeiten in der Hochschulbildung (Kerres 2016). Dieser Veränderungsprozess umfasst die Entwicklung neuer Infrastrukturen und die zunehmende Nutzung digitaler Medien und Technologien, aber auch die Notwendigkeit für Studierende und Mitarbeitende, neue Kompetenzen für ihre derzeitigen und zukünftigen Arbeitsplätze sowie eine gesellschaftliche Teilhabe zu entwickeln.

Dieser Beitrag baut auf der Erfahrung der Arbeit des Hochschulforums Digitalisierung (HFD) auf, das seit 2014 Hochschulen, Hochschulmitarbeitende, Lehrende und Lernende und weitere Akteurinnen und Akteure bei der produktiven Auseinandersetzung mit der Digitalisierung in Studium und Lehre begleitet ${ }^{1}$

\footnotetext{
1 Das Hochschulforum Digitalisierung (HFD) orchestriert den Diskurs zur Hochschulbildung im digitalen Zeitalter. Als zentraler Impulsgeber informiert, berät und vernetzt es Akteurinnen und Akteure aus Hochschulen, Politik, Wirtschaft und Gesellschaft. Das HFD wurde 2014 gegründet. Es ist eine gemeinsame Initiative des Stifterverbandes für die Deutsche Wissenschaft mit dem CHE Centrum für Hochschulentwicklung und der Hochschulrektorenkonferenz (HRK). Gefördert wird es vom Bundesministerium für Bildung und Forschung (BMBF). Weitere Informationen unter https://hochschulforumdigitalisierung.de/de/wir/das-hochschulforum.
}

๖ Open Access. () 2020 Florian Rampelt und Barbara Wagner, published by De Gruyter. @) BY-NC-ND This work is licensed under the Creative Commons Attribution-NonCommercial-NoDerivatives 4.0 License. 
und zu unterschiedlichen Schwerpunktthemen über 50 Arbeits- und Diskussionspapiere veröffentlicht hat. ${ }^{2}$ Zum Abschluss seiner ersten Förderphase stellte das HFD bereits im Jahr 2016 fest, dass die „Chancen der Digitalisierung in Strategie- und Profilbildungsprozesse von Hochschulen einfließen“ (HFD 2016). Diese Fokussierung auf Chancen der Auseinandersetzung mit der Digitalisierung in Studium und Lehre soll auch diesen Beitrag im Sinne einer kritisch-reflektierten, aber lösungs- und zielorientierten Auseinandersetzung prägen.

Den Rahmen sollen vier übergreifende Thesen bilden:

1. Digitalisierung als strategische Aufgabe an Hochschulen erfordert mehr Mut sowie die Bereitschaft zum Lernen voneinander und Arbeiten miteinander.

Die Hochschule der Zukunft wird eine durch Vielfalt geprägte Hochschule sein, in Bezug auf Lernende, in Bezug auf Lernwelten und ganz besonders auch in Bezug auf Lernwege. Dies erfordert deutlich mutigere Hochschulstrategien und eine Öffnung bestehender Strukturen, die sich in einem gezielten Kollaborationsansatz ausdrückt. Fächer-, institutionen- und länderübergreifende Zusammenarbeit sowie die Bereitschaft zum Lernen voneinander werden zukünftig noch viel stärker als bisher eine Voraussetzung für Innovation und erfolgreiches Handeln sein.

2. Hochschulentwicklung muss Studierende stärker als Mitgestaltende in den Fokus rücken.

Lernende werden in den aktuellen Diskursen und Entscheidungen zur Digitalisierung zu wenig mit eingebunden. Für innovative Ideen und neue Perspektiven gibt es oft weder die Offenheit der Strukturen und Prozesse, noch eine grundlegende Bereitschaft, von den letztendlichen Nutzerinnen und Nutzern her zu denken und zu handeln. Die produktive Einbindung studentischer Perspektiven in die Hochschulentwicklung muss noch stärker fokussiert werden, Studierende müssen als Mitgestaltende von Anfang an mitgenommen werden. Die Initiative \#DigitalChangemaker ${ }^{3}$, aber auch die Studierendenorientierung im Rahmen des Projektes Lernwelt Hochschule (Becker/Stang 2020) haben hier starke Referenzen geschaffen.

3. Innovative Lehre muss sich an konkreten Bedarfen orientieren, egal ob digital oder analog.

Die klassische Lehre hat sich seit Jahrhunderten nicht signifikant verändert. Die digitale Transformation ist daher ein notwendiger Anlass, um den Shift from Teaching to Learning in der Praxis umzusetzen. Sie bedeutet aber

2 Alle Publikationen sind unter offener Lizenz hier abzurufen: https://hochschulforumdigitalisierung.de/publikationen.

3 Weitere Informationen unter https://hochschulforumdigitalisierung.de/de/themen/digitalechangemaker-studentische-zukunfts-ag-zu-hochschulbildung-im-digitalen-zeitalter. 
keineswegs einen Ersatz des produktiven Miteinanders in Präsenz, sondern eine sinnvolle Ergänzung, dort wo es passt und auf konkrete Bedarfe trifft. Der Einsatz digitaler Technologien kann helfen, den Studienverlauf zu flexibilisieren und stärker auf individuelle Bedürfnisse der Lernenden zuzuschneiden. Er sollte aber immer der Prämisse Technology second folgen. Innovative Didaktik ermöglicht innovative Lernräume im Analogen wie im Digitalen.

4. Die digitale Transformation erfordert neue digitale und nicht-digitale Kompetenzen bei allen Beteiligten.

Die Auseinandersetzung mit der Digitalisierung von Lebens- und Arbeitswelten ist nach wie vor durch große Unsicherheit geprägt. Hochschulen müssen es als ihre Aufgabe sehen, Strukturen und Angebote zu schaffen, die alle Beteiligten dazu befähigen, einen informierten und mündigen Umgang mit digitalen Technologien zu pflegen. Neue Tools können erst dann wirkungsvoll eingesetzt werden, wenn auch die hierfür notwendigen neuen Kompetenzen vermittelt wurden. Dies beinhaltet nicht nur neue digitale Schlüsselkompetenzen, sondern etwa auch Reflexions- und Adaptionsfähigkeiten.

Es ist, aufbauend auf den zuvor skizzierten Thesen und der einführenden Definition von „Digitalisierung in Studium und Lehre“, in der Auseinandersetzung mit der zukunftsorientierten Gestaltung von Hochschulbildung wichtig, eine weitergehende begriffliche Einordnung vorzunehmen. Die „digitale Lehre“ oder die „digitale Hochschulbildung“ stellt eine im politischen Diskurs gegebenenfalls notwendige begriffliche Verkürzung beziehungsweise eine „Kurzformel für den zugrundeliegenden Transformationsprozess der Bildungsarbeit“ (Kerres 2016) dar, die den konzeptionellen Grundlagen dieses Sammelbandes und auch des vorliegenden Beitrags nicht gerecht wird. Digitalisierung sollte als ein Bestandteil der Hochschulbildung gesehen werden, der diese aber nicht vollständig übernimmt, womit „das Digitale“ „das Analoge“ ersetzen würde. „Diese Dichotomie verkennt, dass das Digitale sich im Analogen verschränkt.“ (Kerres 2018)

Die Digitalisierung in der Lehre stellt daher eine notwendige Präzisierung dar, die deutlich klar macht, dass digitale Technologien und digitale Inhalte eine Rolle in der Lehre spielen, auch innovative Lehre, aber durch weitere Einflüsse geprägt ist und sein sollte. Gleichzeitig scheint es notwendig, ganz im Sinne von Lernwelten der Zukunft, die Lehre gerade im digitalen Zeitalter grundsätzlich nicht losgelöst von den rahmengebenden Studienbedingungen zu betrachten. Das für diesen Beitrag zugrundeliegende Konzept ist daher eine Digitalisierung in Studium und Lehre, die zukunftsfähige Hochschulbildung 
durch eine Anreicherung innovativer pädagogischer Konzepte und Lernräume mit digitalen Technologien ermöglicht. Dies beinhaltet auch eine Berücksichtigung digitaler Kompetenzen in Curricula der Zukunft neben ebenso notwendigen nicht-digitalen Kompetenzen.

Die Digitalisierung in Studium und Lehre erfordert zusammenfassend eine Auseinandersetzung mit dem gesamten Student-Life-Cycle von der Studienorientierung über studienbegleitende Lehr-Lernprozesse bis hin zur Zeugnisvergabe. Die Digitalisierung in Studium und Lehre ist der Anlass, um Möglichkeiten zu identifizieren und nutzbar zu machen, die digitale Technologien bieten, um das Lernen an und mit Hochschulen moderner und noch stärker an den Lernenden orientiert zu gestalten.

\section{Digitalisierung als strategische Aufgabe gestalten}

\section{Strategien für die Digitalisierung in Studium und Lehre}

Hochschulen sehen übergreifend nicht nur eine größere Notwendigkeit der strategischen Auseinandersetzung mit der digitalen Transformation, sondern formulieren auch eine klare strategische Relevanz der Digitalisierung für die Weiterentwicklung von Studium und Lehre (Gaebel/Zhang 2019; Gilch et al. 2019). Dies ist eingebettet in klare Entwicklungen auf Länder-, Bundes- und europäischer Ebene, die in den vergangenen Jahren zu einer strategischer Gestaltung der Digitalisierung im Hochschulbereich führten (Rampelt et al. 2019; European Commission/EACEA/Eurydice 2018). Politische Akteurinnen und Akteure setzen vermehrt Rahmenbedingungen und nehmen strategische Entwicklungsvereinbarungen vor, die der Digitalisierung eine starke Rolle in der Hochschulentwicklung einräumen. Hochschulen sind daher umso mehr gefordert, sich sowohl mit diesen externen als auch den internen Einflussfaktoren auseinanderzusetzen.

Bei einer Auseinandersetzung mit der Digitalisierung als strategische Aufgabe darf diese nicht zum Selbstzweck verkommen (Watolla 2019). Hochschulen müssen sich zunächst mit ihrem eigenen Profil, ihren übergreifenden Schwerpunktsetzungen und Zielen sowie ihren individuellen Stärken auseinandersetzen. Es gilt, neue Möglichkeiten des digitalen Wandels gezielt für die Erreichung dieser Zielsetzungen und der Weiterentwicklung der eigenen Stärken zu nutzen. Eine aktuelle Erhebung im Rahmen der Arbeit der Expertenkommis- 
sion Forschung und Innovation (EFI) ${ }^{4}$ deutet darauf hin, dass eine Mehrzahl der deutschen Hochschulen diesem Prinzip folgt:

\begin{abstract}
Zum Verhältnis von Digitalisierungs- und Hochschulstrategie ergeben die an den Hochschulen durchgeführten Interviews den Befund, dass die Digitalisierung und entsprechende Strategien vornehmlich als Mittel zur Erreichung hochschulstrategischer Ziele gesehen werden. Nur wenn die Digitalisierung nicht selbst im Vordergrund stehe, sondern diese als Mittel zur Erreichung hochschulstrategischer Ziele eingesetzt werde, sei diese nach übereinstimmender Auskunft der Hochschulen erfolgreich. (Gilch et al. 2019, 75)
\end{abstract}

Das Hochschulforum Digitalisierung hat mit der Peer-to-Peer-Strategieberatung ein wirkungsvolles Instrument geschaffen, um Hochschulen im Prozess einer solchen Auseinandersetzung mit der Digitalisierung in Studium und Lehre zu begleiten und eine zukunftsorientierte Reflektion der eigene Ziele und Handlungsmöglichkeiten zu ermöglichen.

\title{
Peer-to-Peer-Beratung: Akteure auf Augenhöhe begleiten
}

Das Hochschulforum Digitalisierung hat, gefördert durch das Bundesministerium für Bildung und Forschung (BMBF), die Peer-to-Peer-Strategieberatung entwickelt, um Hochschulen bei der Weiterentwicklung ihrer strategischen Ausrichtung zu unterstützen. Die Digitalisierung ist dabei der Anlass für eine Auseinandersetzung mit übergreifenden Fragestellungen der Transformation von Studium und Lehre. Zwischen 2017 und 2020 werden im Bundesprogramm jährlich jeweils sechs Hochschulen beraten (Röwert 2019). ${ }^{5}$

Als besonders passend hat sich hierfür der Peer-to-Peer-Ansatz erwiesen, der einen kollegialen Austausch auf Augenhöhe fördert. Das Angebot des HFD unterstützt Hochschulen und alle Statusgruppen dabei, den digitalen Wandel aktiv mitzugestalten und individuelle Schwerpunkte in der strategischen Auseinandersetzung mit der Digitalisierung in Studium und Lehre zu setzen. Akteurinnen und Akteure an Hochschulen werden durch das Programm dabei begleitet und dazu befähigt, Studium und Lehre strategisch weiterzuentwickeln, angepasst an die übergreifenden Chancen und Herausforderungen der Digitalisierung in Studium und Lehre.

Hochschulen, die an dem Beratungsangebot teilnehmen, sollten sich mit der Digitalisierung in Studium und Lehre als einer Querschnittsaufgabe ausein-

4 Weitere Informationen hier: https://www.bmbf.de/de/expertenkommission-forschung-undinnovation-efi-213.html.

5 Weitere Informationen unter https://hochschulforumdigitalisierung.de/de/strategien-fürhochschulbildung-im-digitalen-zeitalter. 
andersetzen, die in einen Bezug zum jeweiligen Hochschulprofil gestellt wird. Sie müssen entsprechend konkrete Herausforderungen im Kontext von Studium und Lehre benennen, die im Rahmen des Peer-to-Peer-Verfahrens aufgegriffen werden sollen und können.

Dabei ist es entscheidend, dass auch unabhängig vom Schwerpunkt der Digitalisierung klare Ziele für die Weiterentwicklung von Studium und Lehre formuliert und digitale Lösungsansätze dazu passend identifiziert werden. Ein wichtiger Teil der Peer-to-Peer-Beratung ist die übergreifende Verantwortungsübernahme und Partizipation aller relevanten Akteurinnen und Akteure (Lehrende, Studierende, Dekanate, zentrale Einrichtungen, Verwaltung). Daher sollten diese in der Durchführung des Beratungsprozesses sowie der anschließenden (Weiter-)Entwicklung von Hochschulstrategien und Umsetzungsmaßnahmen mit einbezogen werden, um Betroffene zu Beteiligten zu machen.

Wesentliches Element der Peer-to-Peer-Strategieberatung sind die sogenannten Peers. Als Peers fungieren ausgewählte Expertinnen und Experten aus der HFD-Community. Diese begleiten die Hochschulen als Critical Friends kritisch und produktiv durch den Beratungsprozess. Dabei bringen sie ihre Erfahrungen in der Strategie- und Organisationsentwicklung an Hochschulen sowie ihre fundierten Kenntnisse im Bereich der Digitalisierung in Studium und Lehre ein. Die Peer-to-Peer-Beratung hat sich dabei als eine Art Reallabor für eine innovations- und partizipationsorientierte Hochschulentwicklung etabliert.

\section{Schwerpunkte setzen: Strategische Handlungsfelder der Digitalisierung in Studium und Lehre}

Die Digitalisierung bringt, trotz der großen Unterschiede zwischen den Hochschulen, ähnliche Aufgaben und Schwerpunkte für alle Hochschulen mit sich. Die Expertinnen und Experten aus der Community des HFD haben in der Reflexion der ersten Beratungsbesuche gemeinsam mit dem HFD 14 Handlungsfelder erarbeitet, die einen Orientierungsrahmen für die Strategieentwicklung bietet. Die hier aufgeführten Handlungsfelder sind Erkenntnisse aus den ersten beiden Runden der Strategieberatung und werden regelmäßig weiterentwickelt. Die 14 Handlungsfelder wurden auf Vorschlag von Expertinnen der TU Hamburg in Anlehnung an klassische Organisationsmodelle den drei Dimensionen Strategie, Struktur und Kultur zugeordnet (Ladwig/Arndt 2020).

Die im Folgenden dargestellten Handlungsfelder (Abbildung 1) sollen auch in Verbindung mit digital verfügbaren Praxisbeispielen ${ }^{6}$, eine übergreifende

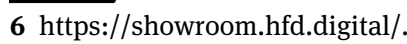


Orientierung für die strategische Auseinandersetzung mit der Digitalisierung in Studium und Lehre geben. Sie sind keineswegs als erschöpfend zu betrachten und sollen stetig weiterentwickelt werden, so wie sich die Anforderungen an die strategische Gestaltung von Studium und Lehre kontinuierlich weiterentwickeln werden.

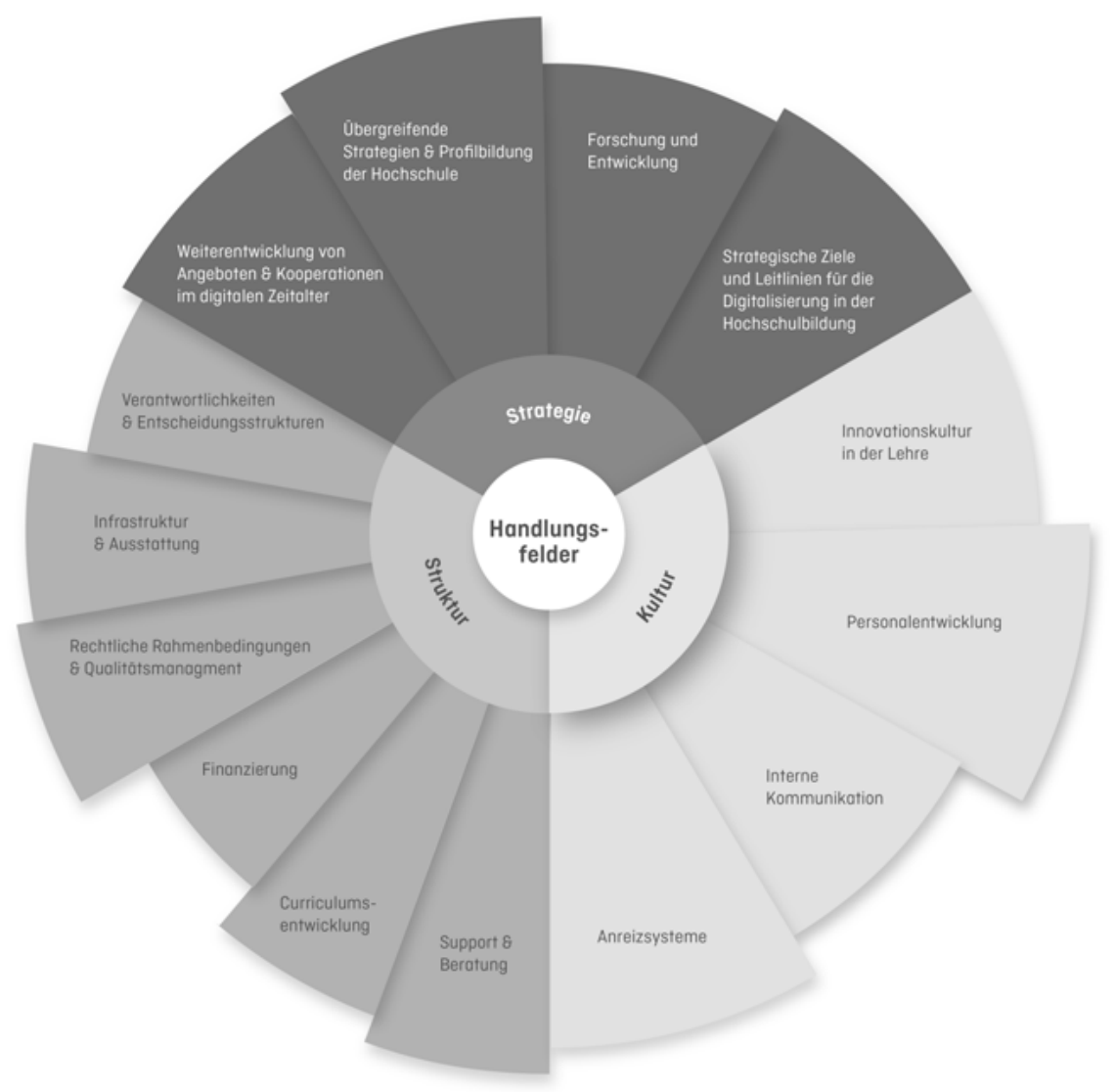

Abb. 1: 14 strategische Handlungsfelder (Eigene Darstellung). 


\section{Strategie}

\section{(1) Profilbildung der Hochschule}

Hochschulen müssen sich im Rahmen von übergreifenden Strategien mit ihrer nachhaltigen Entwicklung und Profilbildung auseinandersetzen. Digitalisierung kann dabei Teil von übergreifenden Strategien sein, die weit über diesen einzelnen Aspekt hinausgehen und vielmehr Synergien unterschiedlicher Schwerpunkte fokussieren. Digitalisierung kann oft dann besonders konstruktiv und produktiv nutzbar gemacht werden, wenn sie als Teil übergreifender Strategien betrachtet wird und damit der Profilbildung der gesamten Hochschule dienlich ist.

\section{(2) Strategische Ziele für die Digitalisierung in Studium und Lehre}

Die richtigen Ziele für die eigene Hochschule zu formulieren und daraus ein Narrativ abzuleiten, ist ein wichtiges Element der Weiterentwicklung von Studium und Lehre. Hierfür kann an Hochschulen auch die Definition von eigenständigen Zielsetzungen zur Digitalisierung in Studium und Lehre hilfreich sein. Solche strategischen Ziele für die Digitalisierung in Studium und Lehre müssen daher klar formuliert und deutlich priorisiert sowie kommuniziert werden. Grundlegend ist hierfür ein klares Verständnis innerhalb der Hochschule, was man unter „Digitalisierung in Studium und Lehre“ versteht. Darauf aufbauend kann etwa ein Mission Statement für die Lehre (im digitalen Zeitalter) auf Ebene der gesamten Hochschule einen Leitrahmen setzen. Die Ableitung von kurz-, mittel- wie langfristigen Maßnahmen auf allen Ebenen der Hochschule kann auch durch einen Leitrahmen für die Weiterentwicklung der digital gestützten Lehre unterstützt werden, aus dem die Fakultäten/Fachbereichen spezifische Ziele und Maßnahmen ableiten können.

\section{(3) Kooperationen im digitalen Zeitalter}

Gerade im Kontext der Digitalisierung sind Kooperationen der Schlüssel, um den digitalen Wandel erfolgreich zu gestalten, bestehende Formen der Zusammenarbeit auszubauen und neue Angebote zu entwickeln. Kooperationen können dabei mit anderen Hochschulen sowie mit außerhochschulischen Akteurinnen und Akteuren geschlossen werden, um hochschuleigene Angebote zu er- 
weitern. Eine zukunftsfähige Hochschule muss es schaffen, Fächergrenzen und institutionelle Grenzen zu überschreiten sowie überregional und international zu kooperieren (Hochschulforum Digitalisierung 2019).

\section{(4) Forschung und Entwicklung}

Auch die Forschung spielt zunehmend eine strategische Rolle in der Weiterentwicklung von Studium und Lehre im digitalen Zeitalter. Im Sinne von Reallaboren können und sollen etwa innovative Ansätze aus Forschungsprojekten unmittelbar auch in der Lehre erprobt und implementiert werden. Dies gilt auf Landes-, Bundes- und europäischer Ebene. Besonders auch das BMBF fördert im Rahmen des Forschungsschwerpunkts „Digitale Hochschulbildung“ in mehreren Förderlinien Forschungs- und Entwicklungsprojekte, die neben einer klaren theoretischen Fundierung und einer wissenschaftlichen Wirksamkeitsforschung auch die konkrete Erprobung in der Praxis ermöglichen sollen. ${ }^{7}$ Dies kann und muss notwendige strategische Impulse setzen.

\section{Struktur}

\section{(5) Verantwortlichkeiten und Entscheidungsstrukturen}

Für die Weiterentwicklung der Hochschulbildung im digitalen Zeitalter ist die Verzahnung von Top-down- und Bottom-up-Prozessen durch klare Verantwortlichkeiten sowie nachhaltige Entscheidungsstrukturen und Partizipationsmöglichkeiten unerlässlich. Neben dem Commitment der Hochschulleitung durch eine aktive Gestaltung der strategischen Entwicklung, muss die Hochschule dazu nachhaltige Entscheidungsstrukturen zwischen der Hochschulleitung und den Fakultäten/Fachbereichen aufbauen sowie Verantwortlichkeiten auf den unterschiedlichen Ebenen festlegen. Wichtig ist, dass eine nachhaltige Fortführung der Strategieentwicklung und -umsetzung auch bei personellen Veränderungen, insbesondere in der Hochschulleitung, durch von Personen losgelöste Rollenbeschreibungen sichergestellt ist. Bei der Strategieentwicklung sind darüber hinaus weitestgehend alle Statusgruppen sowie die zentralen Einrichtun-

7 „Digitalisierung kann dazu beitragen, die Hochschulbildung noch offener, gerechter, internationaler und leistungsfähiger zu machen. Wie kann das am besten gelingen? Das zu erforschen ist Aufgabe des Forschungsschwerpunkts ,Digitale Hochschulbildung“. " https://www. bmbf.de/de/digitale-hochschullehre-2417.html. 
gen und für Studium und Lehre verantwortlichen Stellen der Verwaltung bedarfsgerecht einzubeziehen. Das HFD hat insbesondere auch sehr gute Erfahrungen damit gemacht, Lehrende und Lernende in den Mittelpunkt zu stellen und gerade auch Studierende als „Digitale Changemaker“ mit in die Verantwortung zu nehmen (Böckel 2020).

\section{(6) Finanzierung}

Damit der digitale Wandel in der Hochschulbildung gelingen kann, müssen nachhaltig ausreichend personelle wie finanzielle Ressourcen zur Verfügung gestellt werden. Die Finanzierung ist im Zusammenspiel von Hochschulleitung und Fakultäten/Fachbereichen durch eine nachhaltige Finanzplanung abzusichern. Gerade für kleinere Hochschulen kann dies auch durch die Zusammenarbeit mit anderen Hochschulen ermöglicht werden, indem eine gemeinsame Infra- und Supportstruktur entwickelt und genutzt wird.

\section{(7) Infrastruktur und Ausstattung}

Ein Handlungsfeld zur Gestaltung des digitalen Wandels ist das Vorhandensein einer entsprechenden technischen Infrastruktur: Jenseits von flächendeckenden Breitbandanschlüssen und einer gut funktionierenden WLAN-Ausstattung ist die Verfügung über moderne Hard- und Softwarelösungen entscheidend, um digitale Anwendungs- und Einsatzszenarien zu realisieren. Der Aufbau sinnvoller technischer Infrastrukturen erfordert einerseits signifikante finanzielle Investitionen durch die Hochschulträger und andererseits eine kluge und nachhaltige Auswahl von Lösungen, die insbesondere Synergien in den Hochschulstrukturen berücksichtigen, diese ermöglichen und auf die strategische Hochschulentwicklungsplanung ausgerichtet sind. Der Aufbau von technischen Lerninfrastrukturen ist insofern eine zentrale Aufgabe des strategischen Hochschulmanagements.

\section{(8) Support und Beratung}

Effektive und effiziente Support- und Beratungsangebote können zentral für den Studienerfolg sein. Daher müssen sich auch solche Angebote mit dem digitalen Wandel entsprechend strategisch weiterentwickeln. Gerade im Kontext des digitalen Wandels von Studium und Lehre sind förderliche Rahmenbedingungen 
auch dafür entscheidend, ob Lehrende und Studierende neue Formate, Plattformen, Konzepte und Werkzeuge nutzen. Gerade unerfahrene Lehrende brauchen eine zuverlässig verfügbare mediendidaktische Begleitung durch professionelle Serviceeinrichtungen.

\section{(9) Rechtliche Rahmenbedingungen und Qualitätsmanagement}

Hochschulen sollten die vorhandenen förderlichen Rechtsrahmen der Länder mutiger nutzen, um den Einsatz neuer Lehr- und Lernmethoden und die Weiterentwicklung der Curricula zu fördern. Gerade aber die Nutzung und Produktion digitaler Medien in der Lehre unterliegt großen Unsicherheiten hinsichtlich der rechtlichen Rahmenbedingungen und möglicher Konsequenzen, etwa in Bezug auf das Urheberrecht, Datenschutz oder Kapazitätsverordnungen. Hochschulen tragen dabei eine besondere Verantwortung dafür, dass für alle Mitarbeitenden Rechtssicherheit gilt, gleichzeitig sollten sie den bestehenden Rechtsrahmen aber auch produktiv nutzen. Dies bedeutet auch neue Möglichkeiten zur Anrechnung innovativer, digital gestützter Lehre auf das Lehrdeputat. Hier müssen nicht nur Hochschulen selbst, sondern auch Hochschul- und Wissenschaftspolitik noch deutliche Verbesserungen schaffen.

Gleichzeitig muss die (Weiter-)Entwicklung von Studium und Lehre für das digitale Zeitalter als strategisches Thema auch einem strukturierten Qualitätsund Prozessmanagement unterworfen werden.

\section{(10) Curriculumsentwicklung}

Die Verbreitung digital gestützter Lehr-, Lern- und Prüfungsformen ist ein zentraler Gradmesser dafür, wie breit die Digitalisierung in Studium und Lehre integriert wurde. Um die bedarfsgerechte Umsetzung digital gestützter Lehr-, Lern- und Prüfungsformen und die Nutzung entsprechender digitaler Werkzeuge zu ermöglichen und zu fördern, sollte der Einsatz dieser Formate und Werkzeuge entsprechend in den Studien- und Prüfungsordnungen sowie Modulkatalogen verankert sein. Darüber hinaus ist es erstrebenswert, eine kontinuierliche Weiterentwicklung dieser Aktivitäten zu fördern und Antworten auf die Entwicklung neuer, flexibler Lernpfade zu entwickeln (Orr et al. 2019).

Gleichzeitig bedeutet die Entwicklung neuer Curricula auch die Vermittlung neuer digitaler und nicht-digitaler Schlüssel- und Fachkompetenzen. Unterschiedliche Kompetenzen für das digitale Zeitalter sollten entsprechend studiengangsübergreifender Bestandteil der Curriculumsentwicklung sein. Dazu muss 
sich die Hochschule über für ihre Zielgruppen relevante Kompetenzen und innovative Wege der Kompetenzvermittlung verständigen. ${ }^{8}$

\section{Kultur}

\section{(11) Personalentwicklung}

Personalentwicklung ist ein wichtiger Hebel für die Umsetzung einer Strategie für Studium und Lehre im digitalen Zeitalter. Hochschulen sollten die Spielräume der Personalentwicklung ausnutzen, um den digitalen Wandel in der Lehre voranzutreiben. Dies geschieht einerseits über die Berücksichtigung des Themas bei Berufungsverfahren und dem Ausbau entsprechender Weiterbildungsangebote für Lehrende wie auch über die Erweiterung der Personalstruktur durch wissenschafts-unterstützendem Personal (z. B. Mitarbeitende in Medienund Didaktikzentren, Instruktionsdesignerinnen und -designer etc.). Einen innovativer Ansatz zur Stärkung von innovativer Lehre im Kontext der Personalentwicklung stellt das HFDcert dar, das ausgehend vom Netzwerk für die Hochschullehre des Hochschulforums Digitalisierung gute und innovative Lehre besser sichtbar machen und eine community-basierte Zertifizierung neuer Kompetenzen ermöglichen soll. ${ }^{9}$

\section{(12) Anreizsysteme}

Im Rahmen des kulturellen Wandels an Hochschulen ist es notwendig die Akzeptanz für neue Lehr-, Lern- und Prüfungsformate bei vielen Lehrenden zu erhöhen und diese $\mathrm{zu}$ motivieren, entsprechende Formate auch in der eigenen Lehre einzusetzen. Daher müssen Hochschule attraktive Anreizformate für den Einsatz solcher innovativen Lehr-, Lern- und Prüfungsformaten bieten. Anreize können einerseits dadurch geschaffen werden, dass die Reputation von digitaler Lehre erhöht wird und die Lehrenden in Entscheidungsprozesse eingebunden sind. Zugleich spielen monetäre Anreizstrukturen eine wichtige Rolle. Ergänzend können als Zeichen der Wertschätzung aber auch nach außen sichtbare Auszeichnungen vergeben werden.

\footnotetext{
8 Ein Beispiel für die hochschulübergreifende Vermittlung von Querschnittskompetenzen stellt etwa das durch das BMBF geförderte Pilotprojekt „KI-Campus - die Lernplattform für Künstliche Intelligenz" dar. https://ki-campus.org/.

9 https://hochschulforumdigitalisierung.de/de/hfdcert.
} 


\section{(13) Interne Kommunikation}

Um eine breitenwirksame Integration digitaler Medien in Studium und Lehre zu bewirken, sind die damit verbundenen Ziele und Maßnahmen gegenüber den unterschiedlichen Statusgruppen innerhalb der Hochschule zweckmäßig zu kommunizieren. Hochschulmitarbeitende sind dabei passiv wie proaktiv über technische Lösungen zum Einsatz in Lehre, entsprechende Unterstützungsinfrastrukturen sowie Anreizsysteme zu informieren.

\section{(14) Innovationskultur in Studium und Lehre}

Soll die Einführung und Umsetzung von digitalen Lernumgebungen nachhaltig wirken, so sollten die Lehrpersonen für etwas gewonnen werden, wozu sie zunächst einmal nicht verpflichtet sind: ihre Lehrgewohnheiten zu verändern (z. B. von einem dozierenden zu einem unterstützenden Lehrstil), neue elektronische Prüfungsformen auszuprobieren, Lehrveranstaltungen längerfristig in Kooperation mit externen Stellen vorzubereiten, wenn die Lernressourcen über eine Lernplattform bereitgestellt werden - zumal das Handeln der Lehrenden gegenüber Außenstehenden dadurch transparenter wird. Von Seiten der Hochschule, begonnen bei der Hochschulleitung, sind daher innovative Räume und Lösungen zu schaffen, die kulturelle Veränderungsprozesse unterstützend einleiten.

\section{Nachhaltige Strukturen schaffen und Hochschulen als innovative Lernwelten stärken}

Die Digitalisierung in Studium und Lehre muss an Hochschulen als Chance begriffen und aktiv mitgestaltet werden. Es wurden in den vergangenen Jahren auf unterschiedlichen Ebenen Angebote geschaffen, um diese Gestaltung gemeinsam anzugehen. Das Hochschulforum Digitalisierung hat, als Ergänzung zu zahlreichen Landesinitiativen, länderübergreifend Strukturen zur thematischen Orientierung, zum Austausch zu innovativer Lehre und zur strategischen Auseinandersetzung mit der Digitalisierung in Studium und Lehre pilothaft aufgebaut.

Es zeigt sich gleichzeitig, dass viele Innovationen bereits an einzelnen Hochschulen verfügbar sind und Reallabore, die notwendigen Erkenntnisse 
und Vorarbeiten für übergreifende, innovative Lernwelten schaffen. Um Hochschulen jedoch in der Breite als innovative Lernwelten zu stärken, bedarf es noch weiterer Anstrengungen und nachhaltiger Strukturen, die gewohnte Grenzen überschreiten und die Kollaboration in den Mittelpunkt stellen. Innovative Inseln müssen $\mathrm{zu}$ nachhaltig wirksamen Strukturen weiterentwickelt werden. Dies erfordert, in Anlehnung an die erste These zu Beginn, auch die Bereitschaft zum Lernen voneinander und dazu, das anderswo erfolgreich Erprobte zu kopieren beziehungsweise gemeinsam weiterzuentwickeln, anstatt einmal mehr das Rad neu zu erfinden.

Auch stellt eine neue Verortung im Kontext flexibler Lernpfade (Orr et al. 2019) eine wichtige Aufgabe dar. Hier bedarf es eines systemischen Kulturwandels, der in Ansätzen bereits greifbar wird, aber noch deutliche Transformationsprozesse erfordert. Die Auseinandersetzung mit der Frage, welche Kompetenzen in Zukunft, unterstützt durch digitale Plattformen, hochschulübergreifend gemeinsam vermittelt werden können, steht dabei erst am Anfang.

Hochschulen als innovative Lernwelten sind offene Bildungseinrichtungen, die ihre Stärke daraus gewinnen, zusammenzuarbeiten. Gleichzeitig sind sie offen im Sinne einer Neufokussierung an den Bedarfen, den Lernpfaden und den Mitgestaltungsmöglichkeiten Studierender. Vereinzelte innovative Ansätze müssen hierfür strukturell integriert werden und auch bestehende (hochschulpolitische) Vertretungsmöglichkeiten mit in den Blick genommen werden. Jede Hochschule sollte ihre eigenen digitalen Changemaker haben. Wie mit einem Fokus auf Studierende erprobt, kann dies auch eine Neuorientierung an den Kompetenzen und Bedarfen von Lehrenden ermöglichen. Diese benötigen neue Strukturen zur Innovationsgestaltung sowie der Vermittlung und Sichtbarmachung von künftig notwendigen Kompetenzen. Neue Anforderungen und Rollenbilder können nicht Top-down vorgegeben werden, sondern müssen gemeinsam mit den Lehrenden entwickelt und strukturell gestärkt werden. Future Skills bedeuten entsprechend eine Befähigung zur beruflichen und gesellschaftlichen Teilhabe, die eine strukturelle Veränderung ermöglicht, die von allen gemeinsam getragen wird.

\section{Literatur}

Becker, A.; Stang, R. (Hrsg.) (2020): Lernwelt Hochschule. Dimensionen eines Bildungsbereichs im Umbruch. Berlin; Boston: De Gruyter Saur.

Böckel, A. (2020): Studentiche Perspektive auf die digitale Transformation der Hochschulen. Strukturen, Vernetzung und Partizipation. In: R. Stang; A. Becker (Hrsg.) (2020): Zukunft 
Lernwelt Hochschule. Perspektiven und Optionen für eine Neuausrichtung. Berlin; Boston: De Gruyter Saur, 141-150.

European Commission/EACEA/Eurydice (2018): The European Higher Education Area in 2018. Bologna Process Implementation Report. Luxembourg: Publications Office of the European Union. https://op.europa.eu/en/publication-detail/-/publication/2fe152b6-5efe-11e8ab9c-01aa75ed71a1/language-en.

Gaebel, M.; Zhang, T. (2018): Trends 2018. Learning and teaching in the European Higher Education Area. Brüssel; Genf: European University Association. https://eua.eu/resources/ publications/757:trends-2018-learning-and-teaching-in-the-european-higher-educationarea.html.

Gilch, H.; Beise, A. S.; Krempkow, R.; Müller, M.; Stratmann, F.; Wannemacher, K. (2019): Digitalisierung der Hochschulen. Ergebnisse einer Schwerpunktstudie für die Expertenkommission Forschung und Innovation. Berlin: Expertenkommission Forschung und Innovation (EFI). https://www.e-fi.de/fileadmin/Innovationsstudien_2019/StuDIS_14_2019.pdf.

Hochschulforum Digitalisierung (2016): The Digital Turn. Hochschulbildung im digitalen Zeitalter. Arbeitspapier Nr. 27. Berlin: Hochschulforum Digitalisierung. https://hochschulforumdigitalisierung.de/sites/default/files/dateien/Abschlussbericht.pdf.

Hochschulforum Digitalisierung (2019): Strategies Beyond Borders. Transforming Higher Education in a Digital Age. Book of Abstracts. Berlin: Hochschulforum Digitalisierung. https://hochschulforumdigitalisierung.de/de/strategies-beyond-borders-conference.

Kerres, M. (2018): Bildung in der digitalen Welt. Wir haben die Wahl. denk-doch-mal.de. Online-Magazin für Arbeit-Bildung-Gesellschaft 2. https://learninglab.uni-due.de/sites/default/files/Kerres_denk-doch-mal.pdf.

Kerres, M. (2016): E-Learning vs. Digitalisierung der Bildung. Neues Label oder neues Paradigma? In: A. Hohenstein; K. Wilbers (Hrsg.): Handbuch E-Learning. 61. Ergänzungslieferung. Köln: Fachverlag Deutscher Wirtschaftsdienst. https://learninglab.uni-due.de/ sites/default/files/elearning-vs-digitalisierung.pdf.

Kultusministerkonferenz (2019): Empfehlungen zur Digitalisierung in der Hochschullehre. Beschluss der Kultusministerkonferenz vom 14.03.2019. https://www.kmk.org/fileadmin/ Dateien/pdf/PresseUndAktuelles/2019/BS_190314_Empfehlungen_Digitalisierung_Hochschullehre.pdf.

Ladwig, T.; Arndt, C. (2020): Digitale Strukturen im sozio-technischen Experimentierfeld. Perspektiven der Technischen Universität Hamburg. In: R. Stang; A. Becker (Hrsg.) (2020): Zukunft Lernwelt Hochschule. Perspektiven und Optionen für eine Neuausrichtung. Berlin; Boston: De Gruyter Saur, 121-131.

Orr, D.; Lübcke, M.; Schmidt, P.; Ebner, M.; Wannemacher, K.; Ebner, M. (2019): AHEAD Internationales Horizon-Scanning. Trendanalyse zu einer Hochschullandschaft in 2030. Berlin: Hochschulforum Digitalisierung. https://hochschulforumdigitalisierung.de/de/news/ ahead-studie-hochschullandschaft-2030.

Rampelt, F.; Orr, D.; Knoth, A. (2019): Bologna Digital 2020. White Paper on Digitalisation in the European Higher Education Area. Berlin: Hochschulforum Digitalisierung. https:// hochschulforumdigitalisierung.de/sites/default/files/dateien/2019-05_White_Paper_Bologna_Digital_2020_final.pdf.

Röwert, R. (2019): Unterstützung von Strategien für Hochschulbildung im digitalen Zeitalter durch Peer-to-Peer-Beratungen. Wie die Schärfung der eigenen Hochschulstrategie für Studium und Lehre im Dialog gelingen kann. In: D. Robra-Bissantz; O. Bott; N. Kleinefeld; K. Neu; K. Zickwolf (Hrsg.): Teaching Trends 2018. Die Präsenzhochschule und die digitale 
Transformation. Münster; New York: Waxmann, 43-48. URN:urn:nbn:de:0111-pedocs179209.

Schünemann, I.; Budde, J. (2018): Hochschulstrategien für die Lehre im digitalen Zeitalter. Keine Strategie wie jede andere!. Arbeitspapier Nr. 38. Berlin: Hochschulforum Digitalisierung. DOI: 10.5281 /zenodo.2592258.

Watolla, A. (2019): Strategische Weiterentwicklung von Studium und Lehre im digitalen Zeitalter: Handlungsfelder und Herausforderungen. Diskussionspapier Nr. 6. Berlin: Hochschulforum Digitalisierung. https://hochschulforumdigitalisierung.de/de/news/diskussionspapier-strategische-weiterentwicklung-von-hochschullehre-im-digitalen-zeitalter. 